\title{
Anthroponotic Cutaneous Leishmaniasis, Kabul, Afghanistan
}

\section{Richard Reithinger, ${ }^{\dagger} \dagger$ Mohammad Mohsen, $\dagger$ Khoksar Aadil, $\dagger$ Majeed Sidiqi, $\dagger$ Panna Erasmus, $\dagger$ and Paul G. Coleman*}

A prevalence survey in Kabul City showed that $2.7 \%$ and $21.9 \%$ of persons have active leishmaniasis lesions or scars, respectively. Incidence of disease was estimated to be $2.9 \%$ (29 cases $/ 1,000$ persons per year; 95\% confidence interval 0.018 to 0.031 ). Disease was associated with age and gender; logistic regression analyses showed significant clustering of cases.

$\mathrm{I}^{2}$ n Afghanistan, the majority of leishmaniasis cases are caused by Leishmania tropica (1), which is transmitted anthroponotically (i.e., humans are the reservoir) by the sandfly (Phlebotomus sergenti) (2). Anthroponotic cutaneous leishmaniasis (ACL) is generally characterized by large and/or multiple cutaneous lesions with a variable tendency to self-cure (3). Because of sandfly exposure, most lesions occur on the face, often leading to severe stigmatization in affected persons (e.g., women with lesions are often deemed unsuitable for marriage or raising children). Anecdotal reports suggest that because of the political instability and destruction of the housing and health infrastructure during the Mojahedin and Taliban regimes, $L$. tropica cases have increased during the past decade, with current World Health Organization (WHO) estimates of 200,000 ACL cases in Kabul alone (4). Also, because of several factors (the mass migration of L. tropica-infected [and infectious] Afghan refugees; the sporadic treatment of ACL cases by WHO and nongovernmental organizations; and the virtual absence of vector-control strategies), $L$. tropica has spread to areas where ACL was previously nonendemic (e.g., northwestern Pakistan) (5).

\section{The Study}

Before developing an ACL-control strategy, we conducted a cluster-based, house-to-house survey in Kabul City between July and September 2001 to collect data on the extent of ACL. The leishmaniasis transmission season is between April and October. Each of the city's 14 districts was divided into random clusters according to the

*London School of Hygiene \& Tropical Medicine, London, U.K.; †HealthNet International, Peshawar, Pakistan district's population size, for a total of 90 sample clusters; 30 neighboring households were surveyed in each cluster, with the first household selected at random. A team of medical staff diagnosed disease in household members on the basis of presence or absence of ACL lesions or scars, number of lesions, and date of lesion onset; members were interviewed to collect demographic data such as gender and age. Because of logistic constraints, parasitologic diagnosis of ACL lesions (e.g., microscopic examination or parasite culture) was not carried out. However, in Afghanistan, ACL-like skin lesions from other causes are rare, and our experience suggests that clinical diagnosis has a sensitivity and specificity of $>80 \%$ and $>90 \%$, respectively (Reithinger et al., unpub. data). Written approval to conduct the study was obtained from the Afghan Ministry of Public Health. Informed consent was obtained from study participants; all persons with active cases surveyed were offered free antileishmanial treatment at the HealthNet International clinic.

Of 26,892 persons surveyed, $726(2.7 \%)$ and 5,900 $(21.9 \%)$ had active leishmaniasis lesions or scars, respectively. Of those persons with ACL lesions, the mean lesion number was 2.4 (range 1-50) and the mean lesion duration (to survey date) was 9.1 months (range 0.1-96). A total of 26,887 observations, with full disease and demographic records, from 2,683 households from the 90 sample clusters, were used in logistic regression analyses with a binary outcome variable (ACL lesion or scar).

Four variables were created to assess the distribution of leishmaniasis cases: the prevalence of active lesions in other members of the same household, the prevalence of scars in other members of the same household, the prevalence of active lesions in the nearest neighbor households, and the prevalence of scars in the nearest neighbor households. The nearest neighbor households were defined in terms of the survey protocol. In a given sample cluster, the nearest neighbor to household 1 was household 2; the nearest neighbors to household 2 were households 1 and 3; and so forth. The logistic regressions with robust standard errors (i.e., clustering of households to control for withinhousehold correlations) were also adjusted for age (continuous to year of age at last birthday), gender, and sample cluster (categorical 90 levels). All analyses were conducted in STATA 7 (Stata Corporation, College Station, TX).

The regression analyses show that female patients are at significantly higher odds of having leishmaniasis lesions or scars (Table). Odds of disease were associated with age: elderly people are at slightly greater risk of having active lesions; and elderly persons are less likely to have leishmaniasis scars (the drop in scar prevalence in persons $\geq 12$ years was significant, odds ratio: 0.994 (95\% confidence interval [CI] 0.991 to 0.997$), \mathrm{p}<0.001)$. The analyses showed significant clustering of ACL within and between 
Table. Within- and between-household clustering of anthroponotic cutaneous leishmaniasis lesions and scars, Kabul City, Afghanistan $^{\mathrm{a}}$

\begin{tabular}{|c|c|c|}
\hline \multirow[b]{2}{*}{ Explanatory variables } & \multicolumn{2}{|c|}{ Outcome variable $^{\mathrm{b}}$} \\
\hline & Lesion & Scar \\
\hline Within-household prevalence & $\mathrm{OR}(\mathrm{CI})$ & OR (CI) \\
\hline Prevalence of lesions in other household members & $132.3(67.3$ to 259.8$) \mathrm{p}<0.001$ & 3.728 (2.799 to 4.964$) \mathrm{p}<0.001$ \\
\hline Prevalence of scars in other household members & $1.988(1.500$ to 2.635$) \mathrm{p}<0.001$ & $48.24(41.79$ to 55.68$) \mathrm{p}<0.001$ \\
\hline \multicolumn{3}{|l|}{ Between-household prevalence } \\
\hline Prevalence of lesions in nearest neighbor households & $2.323(0.984$ to 5.486$) \mathrm{NS}$ & $1.585(1.036$ to 1.353$) \mathrm{p}<0.05$ \\
\hline Prevalence of scars in nearest neighbor households & $1.376(0.957$ to 1.980$) \mathrm{NS}$ & 1.184 (1.036 to 1.353$) \mathrm{p}<0.05$ \\
\hline \multicolumn{3}{|l|}{ Other } \\
\hline Age & 1.005 (1.000 to 1.009$) \mathrm{p}<0.05$ & 1.013 (1.011 to 1.015$) \mathrm{p}<0.001$ \\
\hline Sex (female relative to male) & $1.383(1.177$ to 1.626$) \mathrm{p}<0.001$ & $1.186(1.108$ to 1.270$) \mathrm{p}<0.001$ \\
\hline Sampling area $^{c}$ & Chi square $=213.6$, d.f. $=89 \mathrm{p}<0.001$ & Chi square $=330.8$, d.f. $=89 \mathrm{p}<0.001$ \\
\hline Overall model fit & Pseudo $\mathrm{R}^{2}=11.89 \% \mathrm{p}<0.0001$ & Pseudo $\mathrm{R}^{2}=22.72 \mathrm{p}<0.0001$ \\
\hline
\end{tabular}

households (Table). A person's probability of having an active lesion was increased greatly if other lesions appeared on persons in the same household (Figure, A) and also (but less so) if other scars occurred on persons in the same household. A person's probability of having a scar
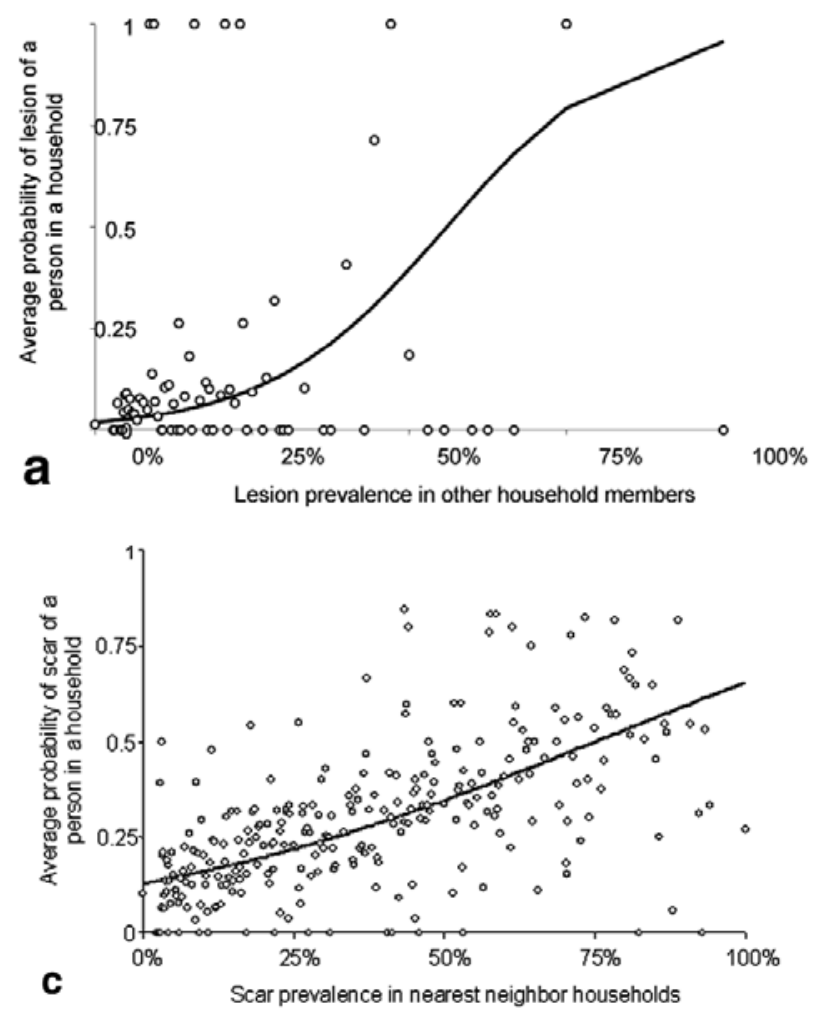

was greatly increased if persons with active lesions or scars were present in the same household (Figure, B). Finally, a person's probability of having a scar was greatly increased with the presence of persons with active lesions or scars in neighboring households (Figure, C). No signif-
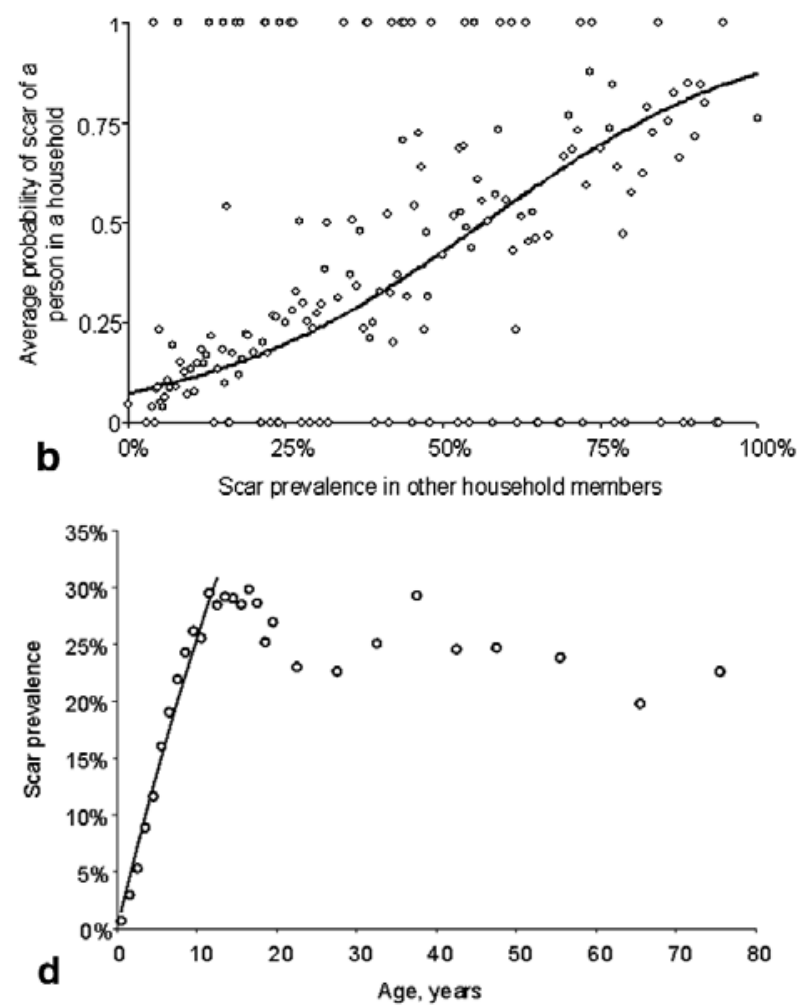

Figure. A, the average probability of having a lesion at different levels of lesion prevalence recorded among other members of the same household (open circles) and the unadjusted fit (solid line) from the logistic regression. B, the average probability of having a scar at different levels of scar prevalence recorded in other members of the same household (open circles) and the unadjusted fit (solid line) from the logistic regression. C, average probability of having a scar at different levels of scar prevalence in nearest neighbor households (open circles) and the unadjusted fit (solid line) from the logistic regression. D, force of infection, $\lambda$, can be estimated from the age-prevalence data, where the proportion, $\mathrm{P}$, of persons with $\mathrm{ACL}$ at age $a$ (where $a$ is age at last birthday plus 0.5 years) is given by $\mathrm{P}(a)=1-\exp (-\lambda a)$ (6). If one assumes that age-independent transmission started 12 years earlier (1), $\lambda$ was estimated by maximum likelihood by using the observed age-prevalence data for children $\leq 12$ y of age. 
icant clustering of ACL lesions occurred between households; however, the sample size was small for analyses. Overall, these findings are consistent with clustering of ACL transmission, including transmission in areas where previous transmission has occurred (because of association with scar prevalence). When maximum likelihood methods (6) are used, the average annual force of ACL infection ( $\lambda$ ) was estimated to be 0.029 per year $(29$ cases $/ 1,000$ persons per year; $95 \%$ CI 0.028 to 0.031 ) over the past 12 years (Figure, D).

\section{Conclusions}

Currently six clinics provide leishmaniasis diagnostic and treatment services in Kabul; an estimated $20 \%$ of the total 67,500 patients (based on the observed prevalence of $2.7 \%$ and a total 2.5 million population for Kabul) are diagnosed and treated. Whether this fact alone could explain the extent and duration of the leishmaniasis epidemic in Kabul is uncertain. Our analyses show that persons are at high risk for active ACL when a high proportion of persons with ACL scars are in the same or neighboring households. The likely explanation for this finding is that sandfly distribution and abundance are patchy but stable over time. The high prevalence of persons with active ACL in Kabul and the comparatively high ACL incidence show that ACL-control strategies (e.g., increasing the number of clinics providing treatment facilities or providing personal protection methods against sandflies) should be conducted soon. We demonstrate that a blanketcoverage ACL-control strategy is not necessary: transmission of this disease is focalized, and interventions (e.g., household insecticide spraying, insecticide-impregnated bednets or chaddars) (5) targeting households with a high proportion of persons with leishmaniasis lesions or scars or city districts containing a high number of high transmission clusters should have a major impact on transmission in Kabul.

The international donor community often considers ACL to be of peripheral importance (e.g., the disease was not included in the basic package of health services for Afghanistan) (7) because this disease has no impact on death rates and patient treatment costs (usually U.S.\$15-200 [8]) are not recovered. Failure to implement a control strategy for this disease will likely lead to an increase in its impact and social stigmatization and represent further problems for a health infrastructure already crippled by 20 years of war.

The opinions expressed by authors contributing to this journal do not necessarily reflect the opinions of the Centers for Disease Control and Prevention or the institutions with which the authors are affiliated.

\section{Acknowledgments}

We are grateful for the logistic support of the Afghan Ministry of Public Health and HealthNet International Khair Khana clinic staff in conducting the survey. We thank Rupert Quinnell for comments on the manuscript.

HealthNet International Malaria and Leishmaniasis Control Program Afghanistan is supported by the European Union, the Gesellschaft für Technische Zusammenarbeit, the Norwegian Afghanistan Committee, the Dutch Government, Thermosurgeries Inc., the United Nations Aid Mission to Afghanistan, and the World Health Organization.

Dr. Reithinger is a program manager for HealthNet International, a Dutch nongovernmental organization that has been working on malaria and leishmaniasis control in Afghanistan for 10 years. His interests are the diagnosis, epidemiology, prevention, and control of infectious diseases, especially leishmaniasis, Chagas disease, and malaria.

\section{References}

1. Ashford R, Kohestany K, Karimzad M. Cutaneous leishmaniasis in Kabul: observations on a prolonged epidemic. Ann Trop Med Parasitol 1992;86:361-71.

2. Killick-Kendrick R, Killick-Kendrick M, Tang Y. Anthroponotic cutaneous leishmaniasis in Kabul: the high susceptibility of Phlebotomus sergenti to Leishmania tropica. Trans R Soc Trop Med Hyg 1995;89:477.

3. Griffiths WDA. Old World cutaneous leishmaniasis. In: Peters W, Killick-Kendrick R, editors. The leishmaniases in biology and medicine. London: Academic Press; 1987. p. 617-43.

4. World Health Organization. Cutaneous leishmaniasis, Afghanistan. Wkly Epidemiol Rec 2002;29:246.

5. Rowland M, Munir A, Durrani N, Noyes H, Reyburn H. An outbreak of cutaneous leishmaniasis in an Afghan refugee settlement in northwest Pakistan. Trans R Soc Trop Med Hyg 1999;93:133-6.

6. Williams B, Dye CM. Maximum-likelihood for parasitologists. Parasitol Today 1994;10:489-93.

7. Trouiller P, Torreele E, Olliaro P, White N, Foster S, Wirth D, et al. Drugs for neglected diseases: a failure of the market and a public health failure? Trop Med Int Health 2001;6:945-51.

8. Boelaert M, Le Ray D, Van Der Stuyft P. How better drugs could change kala-azar control. Lessons from a cost-effectiveness analysis. Trop Med Int Health 2002;7:955-9.

Address for correspondence: Richard Reithinger, Technical Coordinator, Malaria \& Leishmaniasis Control Programme Afghanistan, HealthNet International, 11 - A Circular Lane, University Town, Peshawar, Pakistan; fax: +92 91840 379; email: rreithinger@yahoo.co.uk

All material published in Emerging Infectious Diseases is in the public domain and may be used and reprinted without special permission; proper citation, however, is appreciated. 